\title{
A high-resolution monitoring approach of canopy urban heat island using a random forest model and multi-platform observations
}

\section{Shihan Chen et al.}

Correspondence to: Yuanjian Yang (yyj1985@ nuist.edu.cn)

The copyright of individual parts of the supplement might differ from the article licence. 


\section{S1. Specific inversion steps of related environmental variables}

\section{S1.1 Extraction of IS}

According to related research (Son et al., 2017), Landsat 8 OLI multispectral data will be used to calculate the Normalized Difference Composite Index (NDCI) to extract IS area. The Normalized Difference Built-up Index (NDBI), Soil Adjusted Vegetation Index (SAVI) and Normalized Difference Water Index (NDWI) were calculated. Then NDCI was calculated, and IS area was obtained through k-means clustering. See formula 1-4 for details:

$$
N D B I=\frac{S W I R-N I R}{S W I R+N I R}
$$

Here, SWIR and NIR are the radiation intensity values received in the shortwave infrared band 1 (band 6) and near infrared band (band 5) of Landsat 8 OLI respectively;

$$
S A V I=\frac{(N I R-R)(1+L)}{(N I R+R+L)}
$$

Among them, L is generally selected as $0.25,0.5$ or 1 . When $\mathrm{L}$ is selected as 0.5 , the IS extraction effect is the best.

$$
N D W I=\frac{G-S W I R}{G+S W I R}
$$

$\mathrm{G}$ is the green band (band 3) of the OLI sensor. When the NDWI value is greater than 0.2 , it is considered as a permanent water body and masked.

$$
N D C I=\frac{(N D B I+N D W I) / 2-S A V I}{(N D B I+N D W I) / 2+S A V I}
$$

Since the resolution of the land satellite is 30 meters, an urban pixel is usually composed of IS, water bodies and vegetation. The mixed pixel was converted into a certain pure pixel by fuzzy clustering and threshold method. Then, the distribution of IS was finally obtained.

In the references, the accuracy of IS classification is 0.9. In this study, Taking IS in 2017 as an example, it is compared with the IS produced by Xin Huang et al., and the correct rate is $94 \%$, indicating the relatively high accuracy.

\section{S1.2 Remote sensing indices and surface albedo calculation}

NDVI, gNDVI and SAVI can well reflect the vegetation coverage in the study area and is closely related to temperature changes. The specific calculations of NDVI and gNDVI are as follow:

$$
\begin{gathered}
N D V I=\frac{N I R-R}{N I R+R} \\
g N D V I=\frac{N I R-G}{N I R+G}
\end{gathered}
$$

Among them, $\mathrm{R}$ is the reflectance of OLI red band (band 4). The distribution of Nanjing's NDVI was show in Figure 2d.

NDMI is used to determine vegetation water content. It is calculated as a ratio between the NIR (band 5) and SWIR (band 6) values in traditional fashion:

$$
N D M I=\frac{N I R-S W I R}{N I R+S W I R}
$$


The NDBI uses the NIR and SWIR bands to emphasize manufactured built-up areas. It is ratio based to mitigate the effects of terrain illumination differences as well as atmospheric effects. The calculating method of SAVI and NDBI is shown in Text S1.1.

The air near the surface is generally heated by the long-wave radiation reflected from the surface, so surface albedo has a strong correlation with AT. Surface albedo is the definition of the ratio of reflected emission to the incidence of target object, that is, the ratio of total radiant energy emitted in each direction per unit time and unit area to the total incident radiant energy. This article uses the method of Liang. Landsat8 OLI data were chosen to invert the surface albedo, with the formula being as follow:

$$
\text { albedo }=0.356 B+0.130 R+0.373 N I R+0.085 S W I R 1+0.072 S W I R 2-0.0018
$$

Among them, B is the reflectance of OLI band 2, and SWIR2 is the reflectance of OLI band 7.

In the reference, the quartile of calculated albedo is 5\%. In this study, based on 21 July 2017 albedo, two areas of $1 \mathrm{~km} \times 1 \mathrm{~km}$ water body and vegetation pure pixels are selected to calculate the average, witch are $6.86 \%$ and $15.05 \%$ respectively (both in the normal range).

\section{S1.3 Distance calculation from the city center calculation}

The closer to the city center, the more significant the UHI effect is. Therefore, the distance from it has a significant impact on AT. There are many ways to define the city center. In this article, geometric center was chosen to be the city center. The central formula are as follows:

$$
\bar{x}=\frac{\int x f(x) d x}{\int f(x) d x}, \bar{y}=\frac{\int y f(y) d y}{\int f(y) d y}
$$

Among them, $f(x)$ and $f(y)$ represent the weights of $\mathrm{x}$ and $\mathrm{y}$ respectively, , which are 1 in the calculation of the geometric center. $\bar{x}$ and $\bar{y}$ represent the calculated geometric center coordinates. Then, ignoring the curvature of the earth, the distance were calculated from each weather station to the city center:

$$
\text { dis }=\sqrt{(x-\bar{x})^{2}+(y-\bar{y})^{2}}
$$

Here, $\mathrm{x}$ and $\mathrm{y}$ represent the horizontal and vertical coordinates of the weather station, and dis represents the distance from the city center to the weather station.

\section{S2. Stepwise linear regression and geographically weighted regression}

Stepwise linear regression is the step-by-step iterative construction of a linear regression model that involves the selection of independent variables to be used. In this study, backward selection method was used. its basic steps are: 1 . Start with all explanatory variables in the model. 2 . Remove the variable with the largest P-value, which means the least statistically significant. 3. The new (p-1)-variable model is established and the variable with the largest P-value is removed. 4. Continue until a stopping rule is reached. For instance, stop when all remaining variables have a significant $\mathrm{P}$-value defined by some significance threshold.

A GWR model takes the following form:

$$
Y_{i}=\beta_{0}\left(u_{i}, v_{i}\right)+\sum_{j}^{P} \beta\left(u_{i}, v_{i}\right) X_{i j}+\varepsilon_{i}
$$

where $Y_{i}, X_{i j}$, and $\varepsilon_{i}$ are the dependent variable, the $\mathrm{j}$ explanatory variable (subscripted as $\mathrm{j}$ ), and the random error at point $\mathrm{i}$ (subscripted as subscript i) respectively. The location is denoted by the coordinates $\left(u_{i}, v_{i}\right)$ of a given point $i$. The coefficients $\beta_{j}\left(u_{i}, v_{i}\right)$ are weights function on the location, 
and $\beta_{0}\left(u_{i}, v_{i}\right)$ is the geographical intercept.

\section{S3. Table and caption}

Table S1. band ranges and the main use of Landsat 8 OLI.

\begin{tabular}{|c|c|c|c|c|}
\hline Sensor & NO. & $\begin{array}{l}\text { Wavelength } \\
\qquad(\mu \mathrm{m})\end{array}$ & $\begin{array}{l}\text { Spatial } \\
\text { resolution(m) }\end{array}$ & Usage \\
\hline \multirow{9}{*}{ OLI } & 1 & $0.433-0.453$ & 30 & Coastal monitor \\
\hline & 2 & $0.450-0.515$ & 30 & $\begin{array}{l}\text { Water penetration and discrimination of soil } \\
\text { and vegetation }\end{array}$ \\
\hline & 3 & $0.525-0.600$ & 30 & Vegetation identification \\
\hline & 4 & $0.630-0.680$ & 30 & Observing roads, bare soil and vegetation \\
\hline & 5 & $0.845-0.885$ & 30 & $\begin{array}{l}\text { Biomass estimation and wet soil } \\
\text { identification }\end{array}$ \\
\hline & 6 & $1.560-1.660$ & 30 & $\begin{array}{l}\text { Distinguish roads, bare soil, water and } \\
\text { fog-cloud identification }\end{array}$ \\
\hline & 7 & $2.100-2.300$ & 30 & Rock and mineral identification \\
\hline & 8 & $0.500-0.680$ & 15 & Resolution enhancement \\
\hline & 9 & $1.360-1.390$ & 30 & Cloud detection \\
\hline
\end{tabular}


S4. Figures and captions
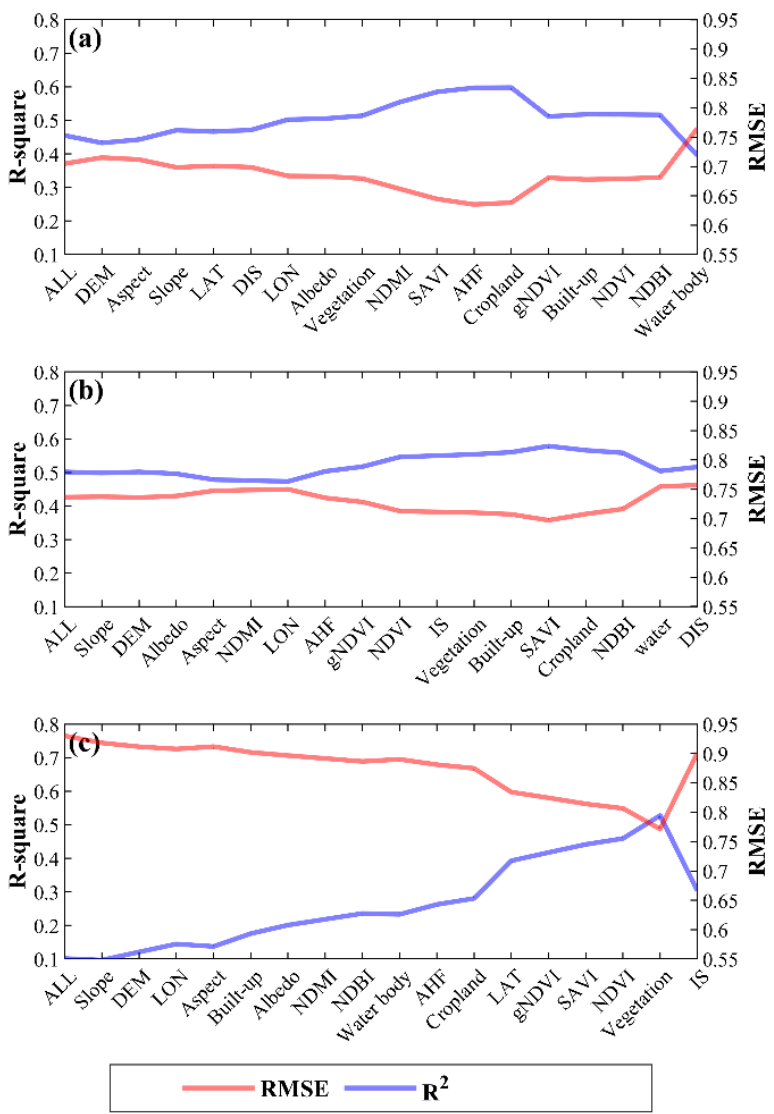

Figure S1. The performance of the RF models under different variable combinations: (a) 11 August 2013; (b) 2 September 2015; (c) 21 July 2017 


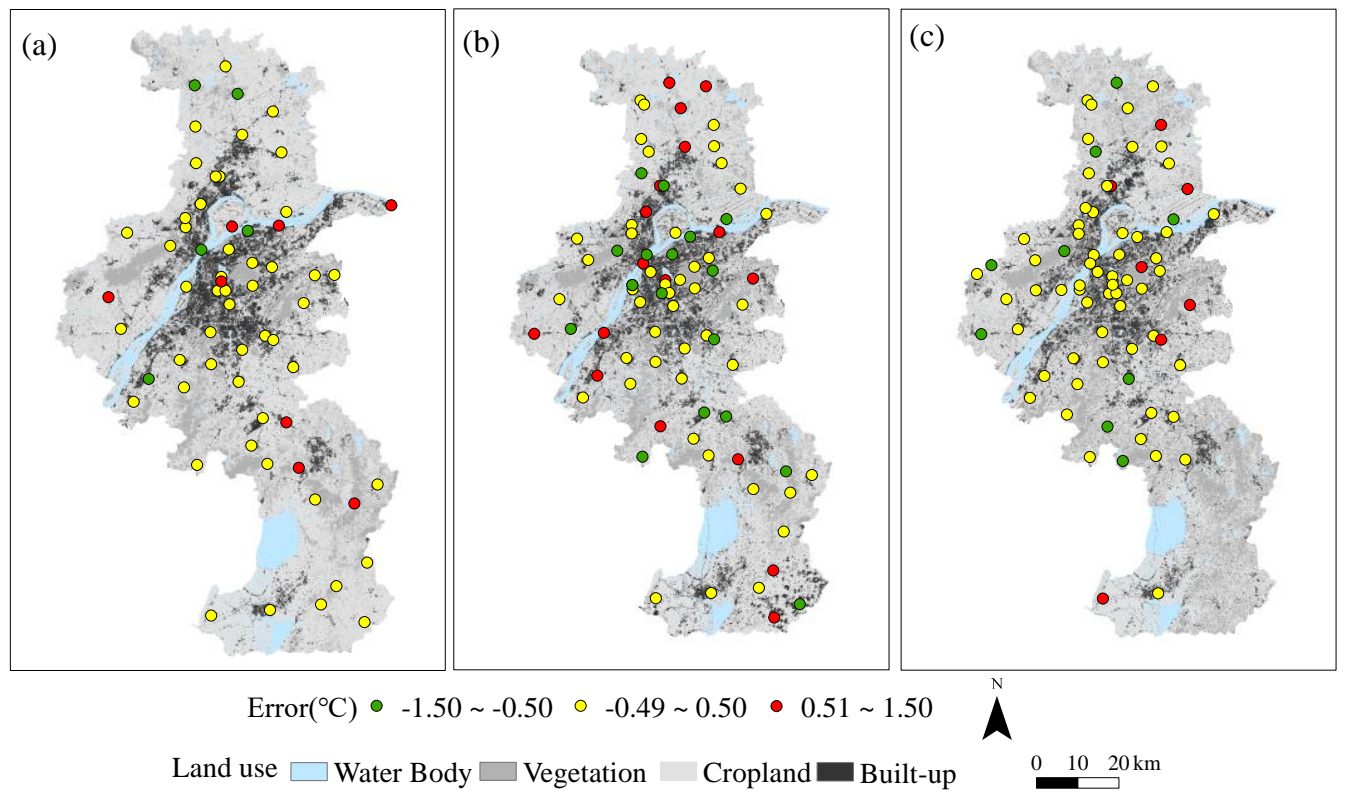

Figure S2. the predicted absolute error of the air temperature(AT) by random forest: (a) 11 August 2013; (b) 2 September 2015; (c) 21 July 2017. 

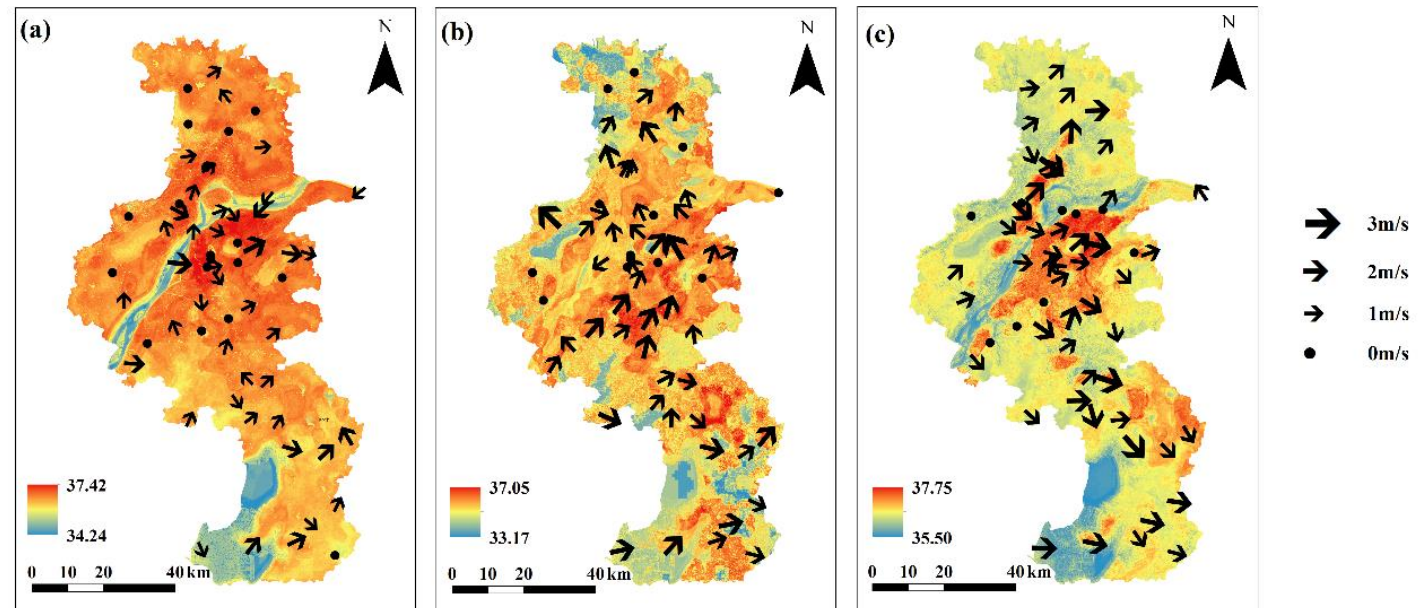

Figure S3. Spatial distribution of air temperature (AT) and wind vector field in Nanjing: (a) 12 August 2013; (b) 13 August 2013; (c) 14 August 2013. 